\title{
Arbeitsunterrichtliche Ansätze für den Geographieunterricht
}

Kurt Bösiger

Unsere Lehramtskandidaten werden heute nach den neuesten didaktisch-methodischen Erkenntnissen geschult und in Theorie und Praxis mit den arbeitsunterrichtlichen Verfahren vertraut gemacht, ja in den Schlußprüfungen von Experten und Examinatoren maßgeblich danach beurteilt, wie sie die Selbsttätigkeit der Schüler anregen und fördern. Dennoch gibt häufig gerade dieser Punkt Anlaß zu negativen Lektionskritiken. Immer wieder muß man beanstanden, daß die Kandidaten zuviel reden, die Schüler dagegen mündlich - und vor allem schriftlich - geistig zu wenig beansprucht werden. Läßt der Methodiklehrer etwa eine im «Dozierstil» abgehaltene Lektion nach arbeitsunterrichtlichen Prinzipien wiederholen oder fortsetzen, so stellt er - meistens in Übereinstimmung mit dem Kandidaten - fest, daß die zweite Methode fast immer produktiver ist. Leider gelingt nicht in jedem Fall eine endgültige Bekehrung, und man erfährt nach relativ kurzer Zeit aus den verschiedensten Quellen, daß gut qualifizierte ehemalige Kandidaten im freieren Schulbetrieb in ihre alten, nicht selten in frühen Vikariaten entstandenen Anfangsfehler zurückgefallen sind. Warum ziehen wohl immer noch viele Lehrer ihr oft überflüssiges Geplauder vor, statt den Kindern ihre Aktivität zu gönnen? Wirkt die eigene Schulzeit so stark nach? Wieviele Lehrergenerationen braucht es, bis sich das Arbeitsprinzip auf breitester Basis durchsetzt? Warum hat sich anderseits in der Geographie der Gedanke der Anschauung so erfreulich Bahn gebrochen? Interessant mag der Hinweis sein, daß nach unsern Erfahrungen der beanstandete Mangel an Aktivierung der Schüler nur für den Schulstubenunterricht Geltung hat. Bei Museumsbesuchen und auf Exkursionen wird die Alleinarbeit der Schüler, sei es einzeln oder in Gruppen, viel spontaner gepflegt. Etwa deswegen, weil die besonderen Probleme des Freiluftunterrichts die Zimmerroutine zum vornherein ausschließen? Ich bin überzeugt, daß diese Feststellungen nicht nur für die Geographie gelten, variiert doch ein redseliger Lehrer das Maß seiner «Dozententätigkeit» kaum nach Fächern.

Im Sinne einer Anregung für jüngere Kollegen seien nachfolgend einige an der Mittel- oder Oberstufe erprobte arbeitsunterrichtliche Ansätze aufgeführt; wie und bei welchen Gelegenheiten sie in den Unterricht eingebaut werden, möchten wir im Hinblick auf unser föderalistisch aufgebautes Schulsy- stem bewußt dem Leser überlassen. Die wenigen skizzierten Möglichkeiten sind als Ergänzung zu meinen vor einiger Zeit publizierten, aber auch im neuen Zusammenhang verwertbaren Beispielen gedacht (vgl. "Zum Problem des Prüfens und Repetierens im Geographieunterricht der Mittel- und der Oberstufe». Geographica Helvetica, H. 1, 1963, S. $98-107)$. Im übrigen begnügen wir uns mit dem Hinweis, daß damit durch einen Lehrer ausnahmsweise auch zwei Klassen gleichzeitig, sei es einzeln oder miteinander im gleichen Raum, nutzbringend beschäftigt werden können: ein billiger individualisierender Unterricht ohne Lehrgerät, falls wirklich einmal Not am Mann ist.

\section{Arbeitsunterrichtliche Ansätze}

\section{Beispiele zur Förderung des Bildungs- und Vor- stellungsgutes}

\section{Erarbeitung ausgewählter Landschaftsgürtel}

[stufenweises Vorgehen]

\section{Atlas- und Buchstudium}

Zuerst gemeinsam, dann einzeln oder gruppenweise Klimacharakter, Vegetation und Landwirtschaft in bestimmten Gebieten studieren. Ergebnisse in entsprechenden Tabellen nach Landschaftsgürteln zusammenstellen:

Tundra, Taiga [eventuell bei Asien einbauen]; Wüste, Steppe, Savanne, tropischer Regenwald [eventuell bei Afrika einbauen].

Voraussetzung: Die gemäßigten europäischen Klimazonen sind im Vorjahr bei der Behandlung Europas erarbeitet worden.

\section{Erkennen der neuen Landschaftstypen}

Mit Hilfe von Diapositiven oder nach «Geographie in Bildern", Bd. 2, Sauerländer: Bilderklärungen mündlich und schriftlich durch die Schüler; Berichtungen, Ergänzungen, Bereinigung der Notizen oder nachträgliche Abgabe der vervielfältigten Lehrerlösung. Repetition eventuell an amerikanischen Beispielen, sofern die Studieraufgaben beim methodisch einfachsten Kontinent Afrika gegeben wurden. 


\section{Vereinfachte Karte der behandelten Landschaf- ten}

Als Schülerskizze oder in Umrißstempel. AnschlieBend Vergleich mit Lehrerlösung [Tafelskizze oder Wachstuchkarte verwenden]. In unteren Klassen eventuell diese Karte oder eine gute Schülerarbeit abzeichnen lassen; in oberen Klassen Ergänzung durch Kausalprofil zweckmäßig.

\section{Schriftliche Arbeit nach Punktsystem}

Am besten unangekündigt, falls man die Gründlichkeit der selbständigen Erarbeitung, die Aufmerksamkeit bei den Diskussionen und die Fähigkeit zum kausalen geographischen Denken überprüfen will.

\section{Geographische Tafelskizze als Rätsel}

Man zeichne beispielsweise den Kanton Graubünden als stumme Karte, wobei durch entwickelnde Darstellung des Umrisses, vielleicht erschwert durch Weglassen von Zwischenstrichen, vorerst das $\mathrm{Ge}-$ biet erraten werden muß. Nachher folgen sukzessive Gewässer, Gebirgszüge, Berge, Pässe, Orte, Verkehr und Wirtschaft. Schülerarbeit mündlich und schriftlich, mit und ohne Hilfe der Karte.

\section{Beispiele zur Erarbeitung oder Festigung geo- graphischer Begriffe.}

\section{Erarbeitung von Begriffen}

Benötigte Begriffe, zum Beispiel über Entstehung und Typen der Vulkane sowie Art der Auswurfmassen, als Disposition an die Tafel schreiben. Hausaufgabe: Definition nach Lehrmitteln und Lexika festhalten. In der Schule: Úberprüfung, Ergänzungen mit Bildbeispielen, später schriftliche Repetition.

\section{Karten- oder Bildauswertung}

Damit alle Schüler beschäftigt sind: vor allem auch schriftliche Auswertung von Spezialkarten im Atlas und vonTypenbildern wie Falaises, Lagunen, Dünen. Frageschema: Was? Warum? Wo? Denselben Begriff womöglich aus Karte und Bild [Diapositive, Schulwandbilder, Bildbände "Geographie in Bildern" bei Sauerländer, Luftphotos] in Aufsatz- oder Stichwortform erarbeiten lassen. Beispielsweise längste und kürzeste Arbeit vorlesen und diskutieren.

\section{Repetition ohne Hilfsmittel}

Die Schüler stellen auswendig ihr Wissensgut in folgender Art zusammen: europäische [außereuropäische] Industriezonen, Verkehrsknoten verschiedener Länder, Kornkammern der Welt, Urwaldgebiete der Tropen und der gemäßigten Breiten, Fremdenorte der Schweiz u. a. m.
III. Beispiele zur Behandlung ausgewählter Landschaften

\section{Bearbeitung von Textblättern}

Der Lehrer erstellt seinen Bedürfnissen entsprechend Textblätter, etwa zur Wirtschaftsgeographie Frankreichs oder Italiens, und vervielfältigt sie. Die Sshüler studieren mittels Atlas selbständig einen in sich geschlossenen zusammenfassenden Abschnitt von 1 bis 2 Seiten. Nach angemessener Zeit, vielleicht schon am Ende der Lektion, stellt der Lehrer schriftlich zu beantwortende Fragen über den wesentlichen Inhalt des studierten Textes. Auch als unangekündigte schriftliche Arbeit möglich, vor allem, wenn Doppelstunden für solche konzentrierte Studierübungen zur Verfügung stehen. Ähnliche Aufgaben sind bei Verwendung des Lehrbuches möglich, doch ist der Lehrer dann an den betreffenden Text gebunden und muß allenfalls das Selbststudium der Schüler mit notwendigen Abklärungen unterbrechen.

\section{Geographischer Vergleich zweier Landschaften in} Tabellenform

Als Ubersichtsbehandlung mit gleichzeitigem Hefteintrag oder als Repetition von zwei einzeln besprochenen Schwerpunktlandschaften geeignet. Alle Hilfsmittel dürfen verwendet werden. Je nach Stufe Stichworthilfen an die Tafel schreiben [zum Beispiel Auswahl aus dem länderkundlichen Schema oder bestimmte Schwerpunkte herausgreifen, etwa Klima, Landwirtschaft und Fischerei], Gelegenheit geben, die Arbeit zu Hause fertigzustellen. Dann einige freie Schülerreferate nach Stichwortzettel an der Wandkarte, womöglich mit Tafelskizzen. Wertung der Referate in der Diskussion. Themen: Norwegen und Schweden, Vorder- und Hinterindien, Argentinien und Brasilien, Sopra- und Sottoceneri.

Entwurf eines Werbeprospektes [nur geographischer Teil]

Als selbständige Arbeit in der Schule oder als Quartalsarbeit, dann aber abgelegenere Landschaften wählen, sonst erhält man schwülstige Abschriften existierender Prospekte!

Wie soll der Text verfaßt sein? Welche Photographien und Schemaskizzen gehören in den Prospekt? Themen: Engadin, Vispertäler, Luganerseegebiet oder aber weniger bekannte Juralandschaften.

\section{Lückentext}

Die Schüler verfassen als Hausarbeit einen geographischen Aufsatz [eventuell Zusammenarbeit mit dem Deutschlehrer], zum Beispiel «Eine Ferienfahrt im Jura». Alle geographischen Namen bleiben offen und werden während des Vorlesens von den Klassenkameraden in Wettbewerbsform erra- 
ten. Jede erste richtige Antwort wird je nach Schwierigkeitsgrad mit 1 bis 3 Punkten bewertet. Unmögliche Antworten, Disziplinlosigkeiten usw. führen zu Abzügen. Auch als Mannschaftswettkampf möglich und beliebt.

\section{Festigung des Nameninventars}

\section{Repetition der Briefträgergeographie}

Als Einzelwettbewerb nach sportlichen Regeln, zum Beispiel Ausscheidungen unter den Banknachbarn. Wer aus einer Dreiergruppe jeweils die erste Antwort weiß, kommt eine Runde weiter. Wer aus der verbliebenen Zweiergruppe das zweite Mal nichts weiß, zu spät oder unsinnig reagiert, scheidet definitiv aus. Im Halbfinal und im Final siegt, wer zuerst 6 oder 10 Punkte erreicht. Je nach Schülerzahl viele Varianten [aufmunternde Hoffnungsrunden] denkbar. Oft gewinnt ein Außenseiter. Mit oder ohne Belohnung [gute Noten]: die Schüler sind stets mit großer Begeisterung dabei. Eventuell zuvor eine "Trainingsstunde» mit Wiederholungskarten von W. Angst (Verlag der Sekundarlehrerkonferenz des Kantons Zürich) oder mit Umrißstempeln ansetzen. Stets soll sich der Lehrer bemühen, wesentliche Fragen zu bringen. Er tritt noch mehr in den Hintergrund, wenn er die Namen an der Wandkarte einfach zeigt [größeres Tempo, konzentriertere Schüleraktivität]. Falls keine stummen Karten vorhanden sind, muß man die Schüler aus den vorderen Bänken versetzen. Auch in einem Wettbewerb erleichtern methodische Hilfen die Aufgabe, beispielsweise durch Einhaltung der Stoffreihenfolge des Lehrplans und Anwendung einer gewissen Systematik beim Abfragen. Etwa so:

1. Schweizer Karte: Gewässer, Gebirgszüge, Orte.

2. Europakarte [physisch]: Grenzverlauf, Relief und Gewässer im Uhrzeigersinn, Hauptstädte und Haupthäfen nach Ländern und Großlandschaften.

3. Weltkarte (physisch): Ozeane und Kontinente, Meere und Inseln. Anschließend über behandelte Erdteile und die besten Schüler auch über nicht besprochene Kontinente abfragen.

An der unteren Oberstufe kann die erst im letzten oder vorletzten Schuljahr zu behandelnde Schweiz auch als Finalthema gewählt werden.

\section{Training und Repetition der Briefträgergeographic}

Ähnlich wie oben, jedoch dieselben Fragen durch die ganze Klasse schriftlich beantworten lassen. Gemeinsame Korrektur nach 30 bis 50 Fragen [Austausch der Blätter]: die richtigen Antworten fortlaufend mit Nummern versehen [Vermeidung von Additionsfehlern]. Ergebnisse des Tests vergleichen; eventuell nur gute Arbeiten zur Aufmunterung bewerten. Periodisch wiederholen, aber nicht in dieser Form als schriftliche Arbeit, da ungleiche Sichtverhältnisse und leichte Betrugsmöglichkeit.

\section{Verwendung von Umrißstempeln}

Briefträgergeographie mit Hilfe des Atlas und einer vervielfältigt abgegebenen Namenliste auf den Umrißstempel eines ausgewählten begrenzten Gebietes eintragen lassen, zum Beispiel Pyrenäen-Halbinsel. Je nach Umfang der Aufgabe Fortsetzung in der nächsten Lektion oder, nachdem alle Namen auf der Karte gefunden wurden, zu Hause fertigstellen lassen. Je nach Zeitbedarf und Schwierigkeitsgrad am Ende der Stunde, in der nächsten oder übernächsten Lektion [meist erst nach vorangegangenem mündlichem Probefragen] unangekündigte schriftliche Arbeit mit Auswahl der wissenswerten grundlegenden Fragen. Leider müssen viele Klassen noch auf der Oberstufe konsequent dazu erzogen werden, gestellte Aufgaben selbständig und intensiv anzugehen und keine Zeit mit "Kunstmalereien» oder andern Nebensächlichkeiten zu verlieren.

\section{Rätsel-Wettbewerb}

Für gruppenweise oder individuell zu beschäftigende Schüler, die nachher ähnliche Rätsel zusammenstellen sollen. Beispiele: Sucht 5 europäische Hauptstädte, deren Anfangsbuchstaben «Basel» ergeben! Lösung: Budapest, Athen, Stockholm, Edinburgh, Luxemburg. Oder: An welchem Fluß liegen folgende Schweizer Städte? Laufen [Birs], Burgdorf [Emme], Langnau [Ilfis], Bischofszell [Thur], Bellinzona [Tessin]. Die Anfangsbuchstaben der Flüsse ergeben, richtig geordnet, ein Hochland in der Alten Welt [Tibet]. 